\title{
Transdermal microconduits by microscission for drug delivery and sample acquisition
}

\author{
Terry O Herndon ${ }^{1}$, Salvador Gonzalez ${ }^{2}$, TR Gowrishankar ${ }^{1}$, \\ R Rox Anderson ${ }^{1,2}$ and James C Weaver* 1
}

\author{
Address: ${ }^{1}$ Harvard-MIT Division of Health Sciences and Technology, Massachusetts Institute of Technology, Cambridge, MA 02139-4307, USA and \\ ${ }^{2}$ Wellman Laboratories of Photomedicine, Dermatology Department, Massachusetts General Hospital, Harvard Medical School, Boston, MA \\ 02114, USA \\ Email: Terry O Herndon - herndon@mit.edu; Salvador Gonzalez - sgonzalez3@partners.org; TR Gowrishankar - tgowrish@mit.edu; \\ R Rox Anderson - rranderson@ partners.org; James C Weaver* - jcw@mit.edu \\ * Corresponding author
}

Published: 19 April 2004

BMC Medicine 2004, 2:12
Received: 14 October 2003

Accepted: 19 April 2004

This article is available from: http://www.biomedcentral.com/l74I-70I5/2/12

(C) 2004 Herndon et al; licensee BioMed Central Ltd. This is an Open Access article: verbatim copying and redistribution of this article are permitted in all media for any purpose, provided this notice is preserved along with the article's original URL.

\begin{abstract}
Background: Painless, rapid, controlled, minimally invasive molecular transport across human skin for drug delivery and analyte acquisition is of widespread interest. Creation of microconduits through the stratum corneum and epidermis is achieved by stochastic scissioning events localized to typically $250 \mu \mathrm{m}$ diameter areas of human skin in vivo.

Methods: Microscissioning is achieved by a limited flux of accelerated gas: $25 \mu \mathrm{m}$ inert particles passing through the aperture in a mask held against the stratum corneum. The particles scize (cut) tissue, which is removed by the gas flow with the sensation of a gentle stream of air against the skin. The resulting microconduit is fully open and may be between 50 and $200 \mu \mathrm{m}$ deep.

Results: In vivo adult human tests show that microconduits reduce the electrical impedance between two ECG electrodes from approximately 4,000 $\Omega$ to $500 \Omega$. Drug delivery has been demonstrated in vivo by applying lidocaine to a microconduit from a cotton swab. Sharp point probing demonstrated full anaesthesia around the site within three minutes. Topical application without the microconduit required approximately 1.5 hours. Approximately $180 \mu \mathrm{m}$ deep microconduits in vivo yielded blood sample volumes of several $\mu \mathrm{l}$, with a faint pricking sensation as blood enters tissue. Blood glucose measurements were taken with two commercial monitoring systems. Microconduits are invisible to the unaided eye, developing a slight erythematous macule that disappears over days.

Conclusion: Microscissioned microconduits may provide a minimally invasive basis for delivery of any size molecule, and for extraction of interstitial fluid and blood samples. Such microconduits reduce through-skin electrical impedance, have controllable diameter and depth, are fully open and, after healing, no foreign bodies were visible using through-skin confocal microscopy. In subjects to date, microscissioning is painless and rapid.
\end{abstract}

\section{Background}

Convenient, cost effective medical technology is needed to provide better care at lower cost. Minimally invasive technologies that meet present and future medical needs 
are extremely desirable. There is, for example, a recognized need for improved diabetes treatment $[1,2]$. With this in mind, minimally invasive transdermal drug delivery and analyte sampling are of long-standing interest.

Molecular transport through the skin is fundamentally limited by the skin's barrier function $[3,4]$. This has motivated the investigation of active molecular and ionic transport through relatively unperturbed skin by diffusion [5] and electrical current (iontophoresis) [6-8]. Other approaches alter the barrier function by using high voltage pulses (electroporation) [9], stress waves [10], or by interventions creating relatively large openings or defects in the stratum corneum. The latter include cavitation by ultrasound $[11,12]$, laser drilled openings [13] and chemically-enhanced electroporation [14]. Microneedles and other sharp devices have also been proposed [15,16]. Of the many techniques investigated, only the hypodermic needle has met the major needs. However, needle introduction is often painful.

An alternative means of avoiding these problems is to create one or more small holes (microconduits) through the stratum corneum and underlying tissues (Figures 1 and 2 ). Techniques using a combination of momentum transfer and scizing are well known in cosmetic dermatology. The relatively hard, roughened stratum corneum and epidermis resulting from aging processes can be removed by moderate velocity, sharp particles impinging obliquely against the skin surface. The hypodermic needle cuts the tissue and holds it open. Upon needle removal, the tissues essentially close the opening. In contrast, microscission can rapidly and painlessly produce small, open microconduits by means of a gas-entrained stream of inert, sharp particles on a defined skin area (Figure 3). We report here on the use of scission through a mask to painlessly produce microconduits in the skin as well as proof-of-concept drug introduction into and analyte extraction from the human body.

\section{Methods}

Here we describe the exploratory use of sharp particles, combined with masking techniques, to define small areas of the skin to be scized. Preliminary experiments were performed using an unmodified Airbrasive Model K, Series II (S.S. White Mfg. Co, Trenton, NJ, USA). Microscopic comparison of various particulates led to the selection of Aluminum Oxide Al-602 (Atlantic Equipment Engineers, Bergenfield, NJ, USA). These fell in the range of $10 \mu \mathrm{m}$ to $70 \mu \mathrm{m}$, with a high percentage of irregular, sharp particles (Figure 4). Parameters such as particle size, shape, velocity, flux, carrier gas pressure and nozzle-mask spacing were varied to investigate their effects on the removal rate of the stratum corneum and underlying epidermis and dermis.
A $75 \mu \mathrm{m}$ thick Teflon mask with one or four holes with a specified diameter and center-to-center distance was used to constrain the area of the skin exposed to the abrasive particles. The mask was mounted on a holder with provision to position the gas nozzle directly above the mask (Figure 5, left and right). The mask with a single $200 \mu \mathrm{m}$ diameter opening, a $450 \mu \mathrm{m}$ diameter nozzle and a nozzle to mask spacing of $1,500 \mu \mathrm{m}$ was used. The particles in a nitrogen stream under a pressure of $552 \mathrm{kPa}$ was directed toward a site on the inner left wrist, $10 \mathrm{~cm}$ back from the center of the palm (Figure 6) of an adult subject. The proof-of-concept results presented in this paper are based on experiments on the research team's principal investigators. The protocol was approved by the Committee on the Use of Humans as Experimental Subjects at MIT. If the mask was held tightly against the skin, the stratum corneum, epidermis and dermis were removed to the capillary level in 20 seconds (Table 1). Blood was clearly visible in the $200 \mu \mathrm{m}$ diameter microconduit, suggesting a depth of 100-150 $\mu \mathrm{m}$. This result led to parameter optimization in five areas.

1. Scizing depends on an optimum incoming particle flux, related to the mask opening diameter. Lower and higher fluxes reduce scizing rates. Excess particles per unit time tend to clog the mask opening and cause particle trapping between the mask and stratum corneum. At this limit, excess particles impede the entry and exit of particles, slowing the scizing process. The particle generator has a pressurized particle reservoir, the bottom of which is separated from the carrier gas path by a thin aperture plate with holes in it. This system is vibrated by an electrical solenoid with motion amplitude control to provide controlled delivery of microparticles into the gas stream. This arrangement was modified by replacing the eight, $500 \mu \mathrm{m}$ diameter holes with a single $325 \mu \mathrm{m}$ diameter opening, giving a particle flux that produced a peak scission rate near the maximum shake amplitude.

2. High gas pressure deflected the stratum corneum away from the mask, causing particle trapping and poor microconduit definition due to loss of particulate collimation. Experiments on test polyethylene substrates that are 'soft' and on human skin in vivo showed the scission rate to be the same at 103 to $172 \mathrm{kPa}$ as at $552 \mathrm{kPa}$.

3. The particle flux was initially observed to vary significantly due to the reservoir shake solenoid, synchronized with valving in the gas stream creating large pressure pulses. This variability was greatly decreased by removing all gas flow controls and externally actuating the reservoir vibration solenoid. A voltmeter was installed in the shake solenoid to permit accurate shake amplitude repeatability. After the gas pressure is turned on and flow established for ten seconds, the shake solenoid is energized. 


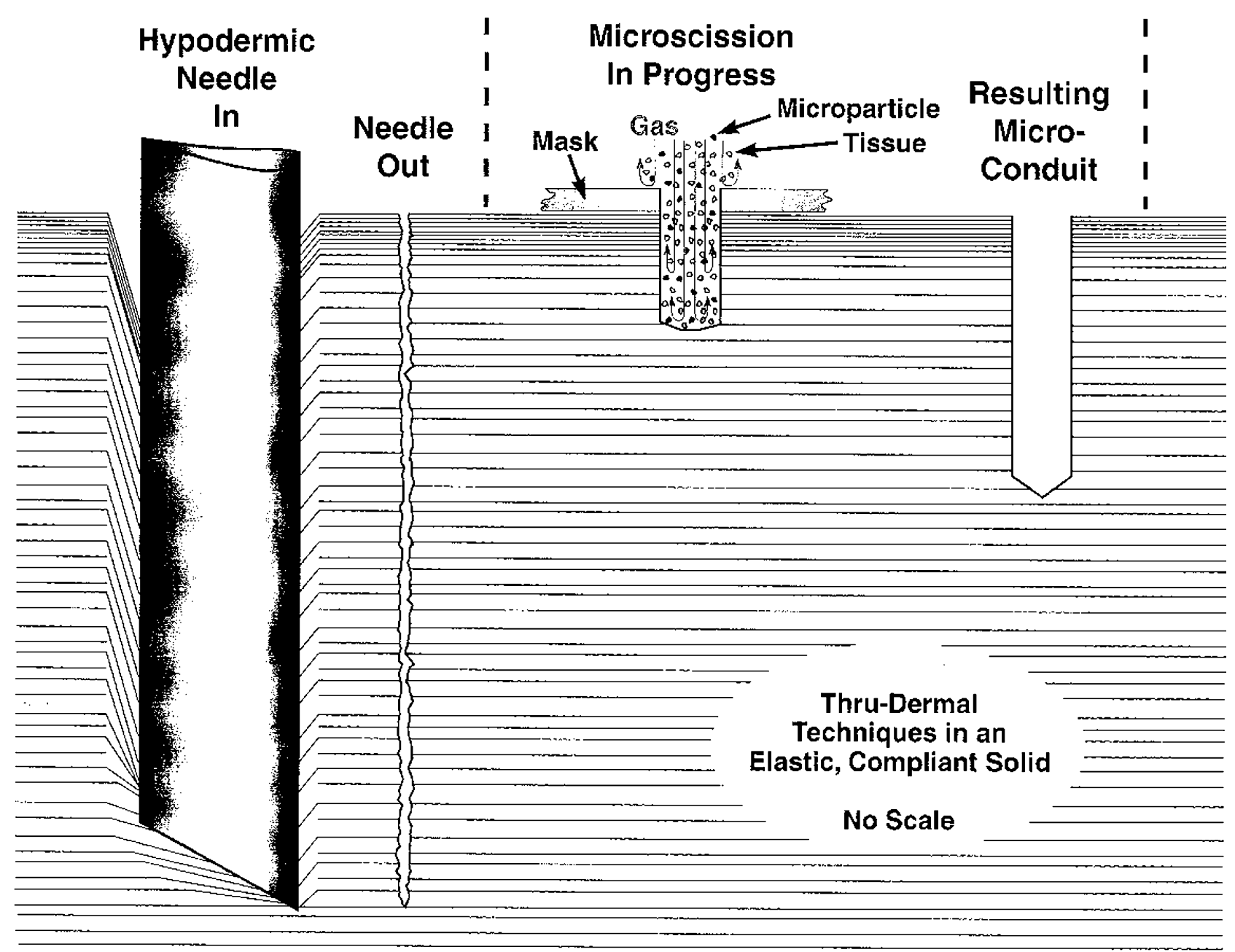

Figure I

Physical effects of hypodermic needle and microconduit.

This sequence is reversed to turn the system off. A full cycle particle flux variation of $4 \%$ is achieved, with the flux during scission constant to within $2 \%$.

4. Carbide nozzles (S.S. White Mfg. Co, Trenton, NJ, USA) with aperture diameters of either $275 \mu \mathrm{m}$ or $450 \mu \mathrm{m}$ were used. Smaller diameters clogged, and larger diameters produced excessively large flux diameters at the mask surface for the single or four hole masks used throughout. A nozzle to mask spacing of $750-850 \mu \mathrm{m}$ produces a 3,800 $\mu \mathrm{m}$ diameter flux cone. This exposes the surface of the stratum corneum to approximately 350-500 particles per second through each $150 \mu \mathrm{m}$ diameter hole in the one or four opening masks at a shake voltage of $74 \mathrm{~V}$ and nitrogen gas pressure of $138 \mathrm{kPa}$. These parameters give a scizing rate of approximately $10-15 \mu \mathrm{m}$ per second on the stratum corneum of adult subjects who participated in the in vivo trials. The mask holder with the four nozzle array below it is shown (Figure 5, left). The mask is cut to extend beyond the circular ring that presses it against the stratum corneum. The square hole that rigidly fixes the nozzle location in three axes is above it. The rectangular arm extending left acts as a bridge over which a strap around the wrist pulls the holder against the wrist. At the left end is a square, electrically insulating plate that acts as a stabilizing fulcrum. The four-hole mask (Figure 5, right) is seen from the stratum corneum side, backed by a thin metal plate with a hole having a $100 \mu \mathrm{m}$ ridge around its edge that presses the mask against the stratum corneum. Three nozzles are just visible through the mask.

5. Polyimide film - a hard, electrically insulating, high temperature polymer - was used for scission rate testing and as a mask material. It exhibited the same resistance to mask thinning by impinging particles as teflon, which scize less rapidly than glass or stainless steel. Polyimide 


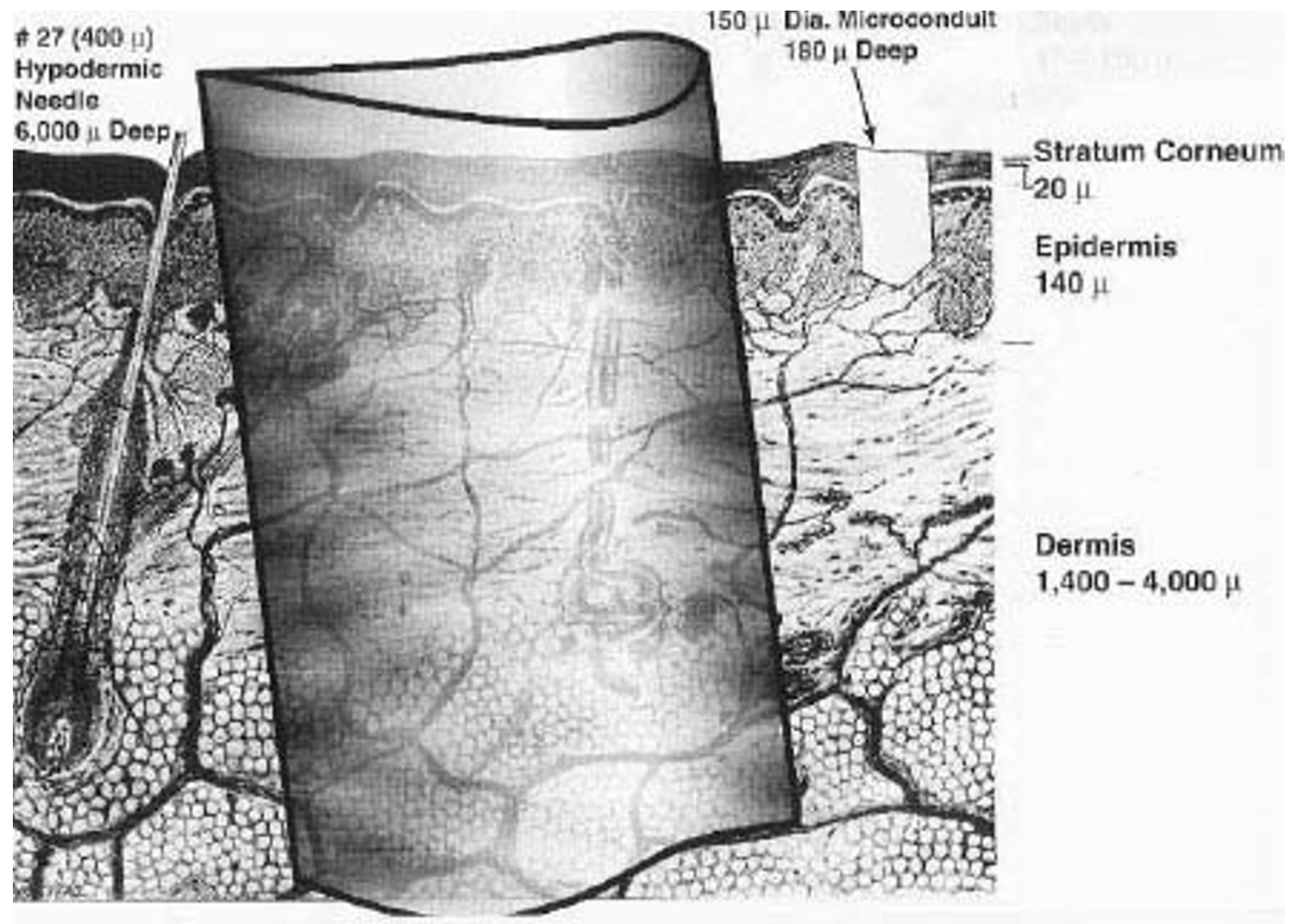

Figure 2

Size comparison of hypodermic needle to a microconduit.

\section{Microscission In Progress}

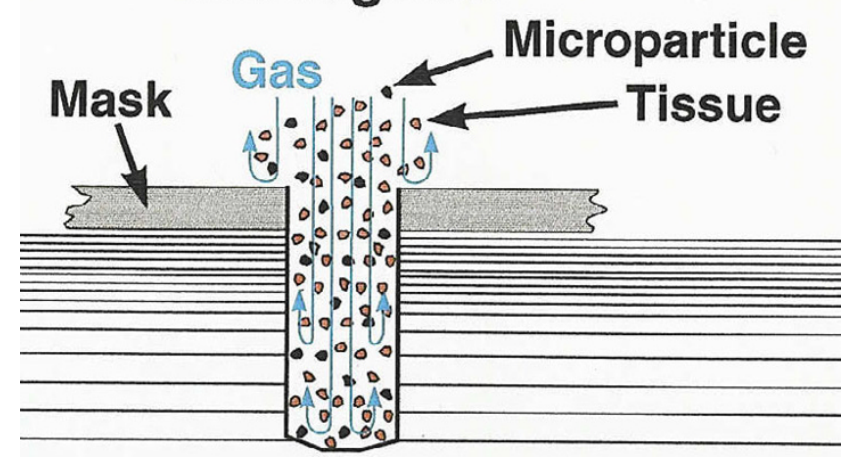

Figure 3

Process of masked scission. can be shaped readily by laser ablation, drilling, chemical or plasma etching, lending itself to low cost, mass produced single-use masks. Polyimide mask life time is 6090 seconds, equivalent to opening five to eight microconduits $150 \mu \mathrm{m}$ deep, providing ample margin for a single scizing operation. Beyond this life time, the masking holes in polyimide increase in size by extended abrasion.

\section{Results and discussion \\ Scized microconduits in vivo}

Attempts to do in vitro testing were abandoned when properties of human cadaver skin were found to be quite different from in vivo skin. There was considerable sample-to-sample variability in the stratum corneum thickness and the skin impedance in human cadaver skin prior to microscissioning. The variability may have been caused by the preservation techniques, length of time between death and skin harvest and the site of skin on the body. Superficial mechanical state of the skin varied from tough to 


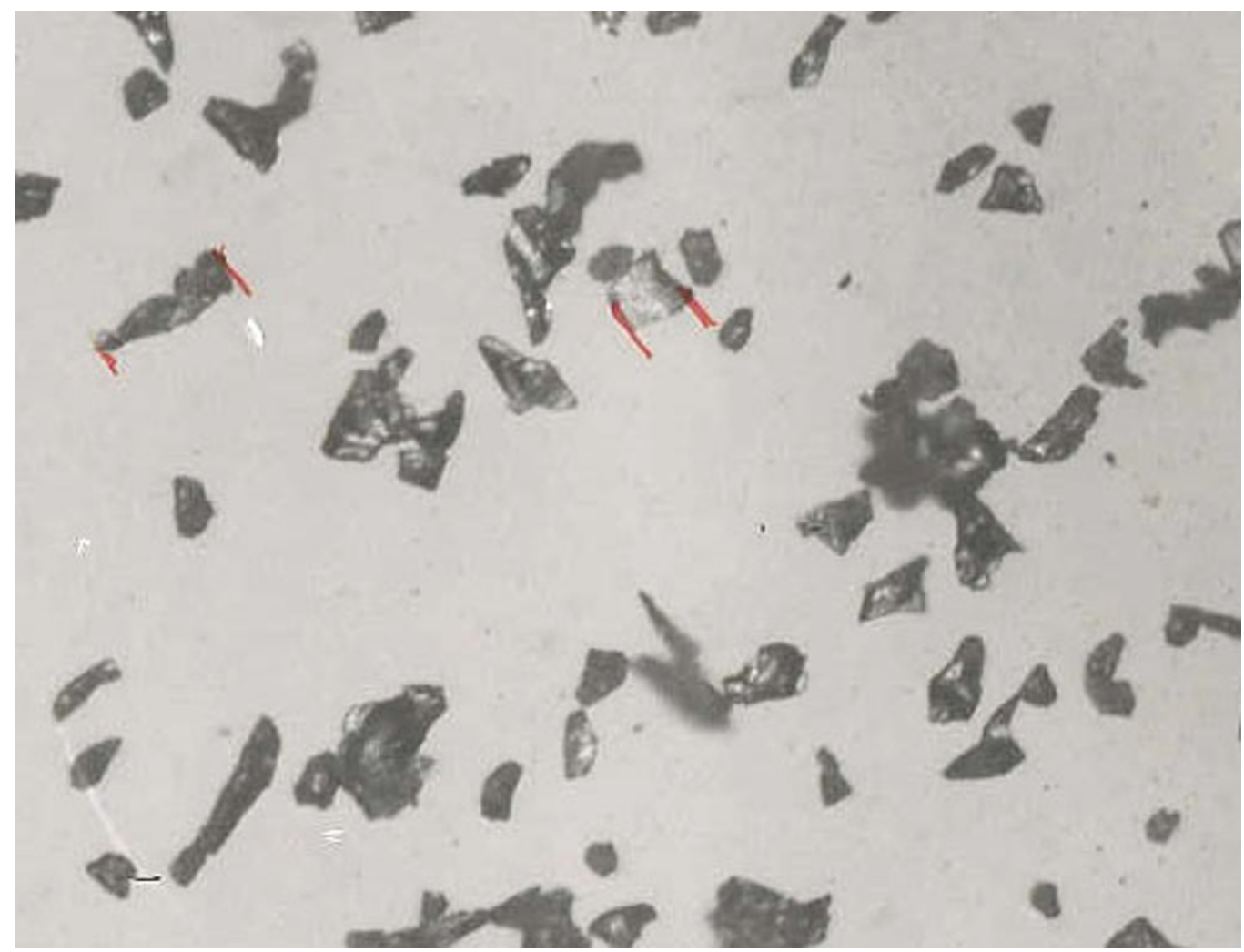

Figure 4

$\mathrm{Al}_{2} \mathrm{O}_{3}$ scizing particulate (marked width upper center $=35 \mu \mathrm{m}$ ).
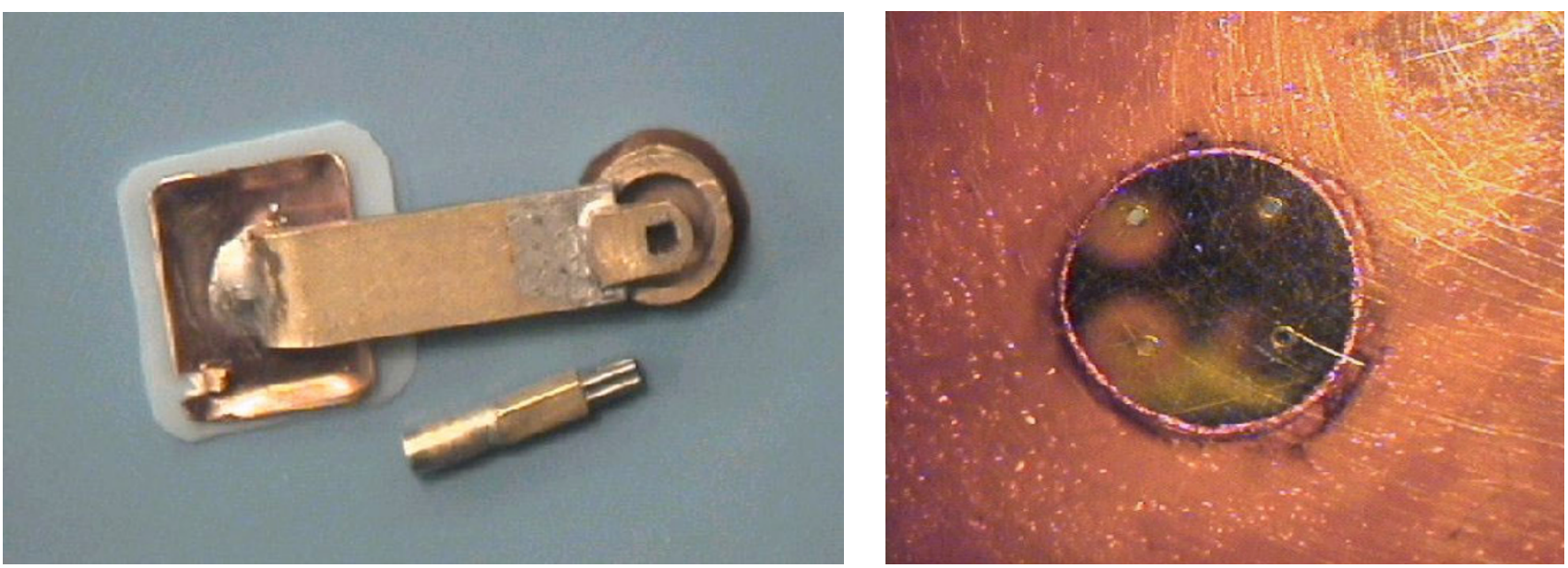

\section{Figure 5}

Mask holder and nozzle fixture: Left: Mask/nozzle holder and wrist fixture; Right: Close up of 4-hole mask and nozzles. 


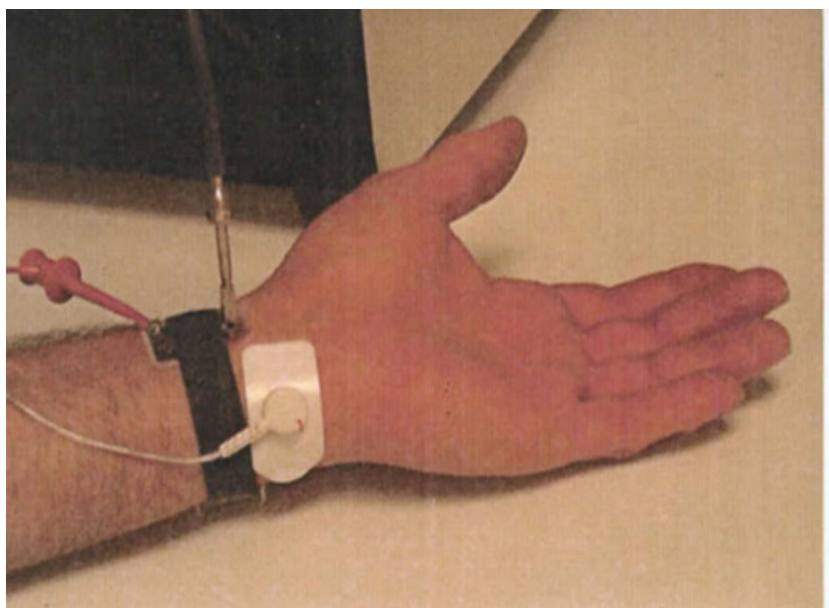

\section{Figure 6}

Microscissioning arrangement: Forearm with attaching strap, mask holder, nozzle, electrical connections to holder and ECG electrode.

near-disintegration among samples. As the wide range of possible applications became apparent, a decision was made to demonstrate proofs-of-concept only. Reliable demonstration of drug delivery (lidocaine), analyte sampling (blood glucose) and even electrical impedance measurement were not possible with in vitro testing.

All in vivo results are from experiments done on two adult subjects. We sterilized the aluminum oxide particulate and masks by heating to $200^{\circ} \mathrm{C}$ for one hour. Nozzles, hoses and the particle reservoir were rinsed in methanol. Sterile gloves were worn and the target areas swabbed with ethanol. Sterile saline solution was used for electrical tests with syringes and needles for handling saline being rinsed with methanol, and nitrogen dried prior to use.

The depth of scized microconduits can be determined approximately by measuring the electrical impedance between an ECG electrode on the subject's unperturbed skin and the mask holding fixture (Figure 6). A SR715 LCR meter (Stanford Research, Sunnyvale, CA, USA) operating at $1 \mathrm{~V}$ and $1 \mathrm{kHz}$ was used. The metal mask holder was electrically connected to the microconduit by normal saline placed in the ring to which the mask was bonded. Since the mask was held tight against the skin, the saline did not leak between the mask and skin, thus providing electrical continuity with the microconduit. Typical microconduits generated with a four nozzle, four hole mask are shown (Figure 7, left and right). The data (Table 1) are averages of approximately 100 experiments on two subjects. Microconduit depths were measured using the Vivascope 1000 (Lucid, Rochester, NY, USA), an in vivo near-infrared reflectance-mode confocal microscope, with an illumination wavelength of $830 \mathrm{~nm}, 30 \mathrm{~mW}$ power and a $30 \times, 0.9$ N.A. water immersion objective providing a viewing depth of $200 \mu \mathrm{m}$. The instrument captures images with a spatial resolution of 0.5 to $1.0 \mu \mathrm{m}$ in the lateral dimension and 3 to $5 \mu \mathrm{m}$ in the axial dimension. Further details of this system have been reported recently [17]. Standard particle generator parameters were used, that is, $\mathrm{N}_{2}$ under $138 \mathrm{kPa}$ pressure, reservoir drive of $80 \mathrm{~V}$, $450 \mu \mathrm{m}$ nozzle diameter.

Microconduit profiles are revealed by infrared confocal microscopy (Figure 8). Microconduits made with a four hole mask and a scission time of 20 seconds are shown on the left. The white lines are an electron microscope grid placed on the skin to provide high contrast for focusing. The $160 \mu \mathrm{m}$ diameter openings are nearly uniform, with the upper right one corresponding to the upper left image in the confocal views. The confocal images at nine depths clearly illustrate that the microconduit is fully open throughout its depth of approximately $165 \mu \mathrm{m}$ (lower right image). At its bottom, the diameter is approximately $65 \mu \mathrm{m}$.

Usually there are no residual $\mathrm{Al}_{2} \mathrm{O}_{3}$ microparticles, which are easy to see as bright 30 micrometer particles at full depth. In some cases, up to about ten microparticles were observed. However, a deionized water rinse from a hypodermic needle at low pressure is effective in removing particles. In cases where particles were seen and left, they were not visible in the confocal microscope at the site after full healing. This suggests they were moved out by the healing process, but has not been confirmed by other methods.

Table I: Microconduit depth and electrical resistance versus scission time.

\begin{tabular}{|c|c|c|c|}
\hline Scission time(s) & Holder condition & Microconduit to ECG resistance & Microconduit depth $(\mu \mathrm{m})$ \\
\hline No scission & Dry & $\mathrm{I}-3 \mathrm{M} \Omega$ & 0 \\
\hline No scission & Saline & $\mathrm{I}-3 \mathrm{M} \Omega$ & 0 \\
\hline $2-5$ & Saline & $100-200 \mathrm{k} \Omega$ & $10-30$ \\
\hline 10 & Saline & $50-70 \mathrm{k} \Omega$ & $30-70$ \\
\hline 15 & Saline & $28-35 \mathrm{k} \Omega$ & $70-100$ \\
\hline 20 & Saline & $18-24 \mathrm{k} \Omega$ & $100-160+$ \\
\hline
\end{tabular}



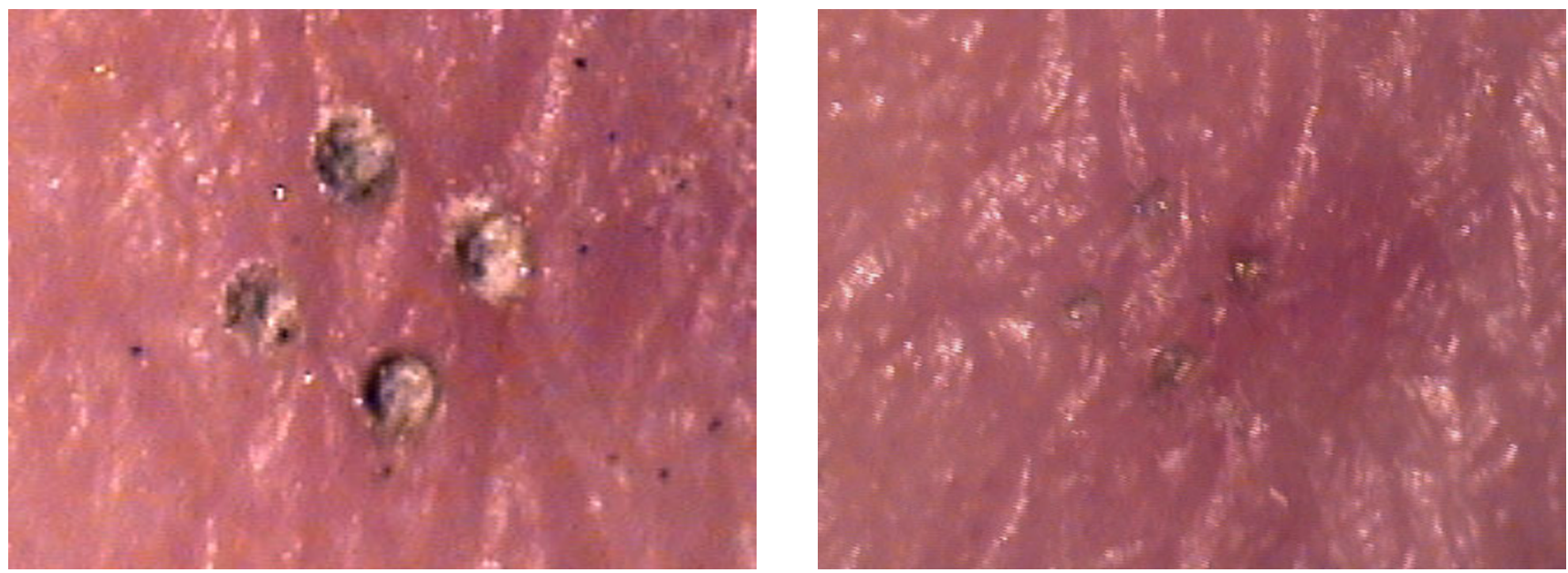

\section{Figure 7}

In vivo microconduits: Four I50 $\mu \mathrm{m}$ microconduits on $450 \mu \mathrm{m}$ centers with abrasive particles present in the microconduits (left) and with particles removed from the microconduits (right).

\section{Transdermal electrical impedance reduction}

Removal of the high electrical resistance stratum corneum takes place during the initial few seconds of scizing a microconduit. If stopped, then scizing is a fast, simple, totally sensation-free method for reducing the electrical impedance through the skin. At $1 \mathrm{kHz}$ and $1 \mathrm{~V}$, the impedance between two electrocardiogram electrodes (Type 510-005, Lynn Medical, MI, USA) is approximately $4 \mathrm{k} \Omega$. Placing two of the same type of electrocardiogram electrodes over two $200 \mu \mathrm{m}$ diameter shallow microconduits reduces the impedance to $500 \Omega$, measured under the same conditions. This implies that the $1 \mathrm{kHz}$ impedance associated with a single microconduit is only $250 \Omega$, and, therefore, four microconduits can provide a local skin resistance of the order of $100 \Omega$ each.

\section{Lidocaine delivery}

Assessment of microconduit efficacy for delivering a drug into the dermis and epidermis was carried out using the topical anaesthetic, lidocaine. The presence of lidocaine was evaluated by using non-scarring pulses from a 585 nm pulsed-dye laser, employed as a pain inducer [18]. Also, the 'pin stick' on skin around the microconduit tests for sensation was used to map lateral anesthesized distance from the microconduit. Masks with four $160 \mu \mathrm{m}$ diameter holes on $450 \mu \mathrm{m}$ centers were used. Testing was done on the inner, left wrist, approximately $10 \mathrm{~cm}$ away from the palm center.

The first test determined lidocaine uptake and level of anesthesia. After scission for 7 seconds, a subject reported a barely perceptible 'pricking' sensation. The microcon- duits had an impedance of $23 \mathrm{k} \Omega$, implying a depth of $140 \mu \mathrm{m}$. A slight discharge of clear fluid was evident. Both the microconduit and control sites were exposed to a 50\% lidocaine solution ( 5 gm lidocaine hydrochloride in $5 \mathrm{ml}$ normal saline) in saturated filter paper pads with Finn chambers taped over each. In prior studies, we used $40 \%$ and 50\% lidocaine solution and found that the subjective degree of anesthesia was easily quantifiable by the subjects enrolled in those studies, so we selected 50\% lidocaine concentration for this proof-of-concept demonstration [18]. These two sites were exposed to a lidocaine 'soak' for 5 minutes. A third 'normal' control site, receiving no lidocaine exposure, was marked and tested. These sites were separated by $5-6 \mathrm{~cm}$. Sensation testing was done with a Coherent Palomar (Burlington, MA, USA) Light Sheer Laser emitting a $30 \mathrm{~ms}, 35 \mathrm{~mJ}$ pulse. The sites were lazed randomly three to five times each, with and without power on, while the subject's arm was extended and the subject looked away. The subject verbally reported sensation effects. The testing was carried out 15, 30 and 75 minutes after lidocaine exposure (Tables 2 and 3). The sensation data strongly suggest that the area around microconduits was anesthetized.

A number of additional tests were done using the 'pin stick' test for sensation, to determine the minimum time for full anaesthetic effect. Most testing was done on one subject, with verification tests done on two other subjects. These tests were done on a single $160 \mu \mathrm{m}$ diameter microconduit, $100-150 \mu \mathrm{m}$ deep on the left inner wrist. Using 50\% lidocaine saturated cotton pads as the anesthetic source, anesthesia of a control area without a micro- 


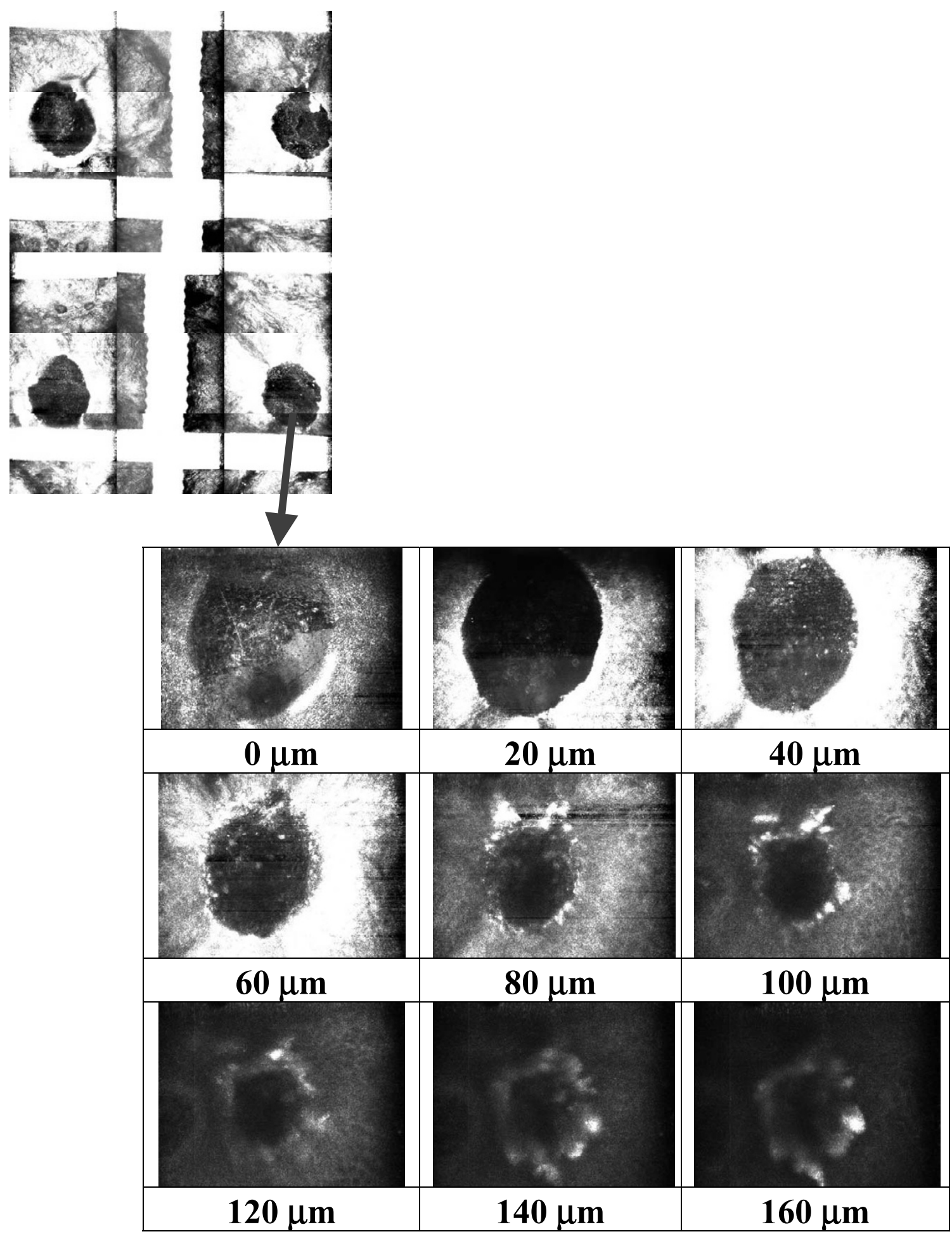

Figure 8

Depth profile of a microconduit: Top: Confocal skin surface view of four $160 \mu \mathrm{m}$ diameter microconduit openings in vivo in human stratum corneum. Bottom: Cross-section of lower right microconduit (blue arrow) with increasing depth obtained by in vivo confocal microscopy. 
Table 2: Efficacy of lidocaine delivery to tissues through four microconduits.

\begin{tabular}{|c|c|c|}
\hline \multirow{2}{*}{$\begin{array}{l}\text { Site treatment Four microconduits, } \\
\text { soaked with lidocaine for } 5 \text { minutes }\end{array}$} & \multicolumn{2}{|c|}{ Sensation description (at two laser powers) } \\
\hline & $35 \mathrm{~mJ}$ & $40 \mathrm{~mJ}$ \\
\hline \multicolumn{3}{|l|}{ I 5 min after lidocaine } \\
\hline Control - no lidocaine, no microconduit & Sharp interior pain & Sharp interior, very painful \\
\hline Control - lidocaine, no microconduit & Sharp interior pain & Sharp interior, very painful \\
\hline lidocaine microconduit site & Hardly perceptible & Hardly perceptible \\
\hline \multicolumn{3}{|l|}{30 min after lidocaine } \\
\hline Repeat I5 minute test sequence & No changes in sensation levels & \\
\hline \multicolumn{3}{|l|}{75 min after lidocaine } \\
\hline Control - no lidocaine, no microconduit & Sharp interior pain & Sharp interior, very painful \\
\hline Control - lidocaine, no microconduit & Sharp interior pain & Sharp interior, very painful \\
\hline lidocaine microconduit site & Considerable pain & Sharp interior pain \\
\hline
\end{tabular}

Table 3: Efficacy of lidocaine delivery to tissues through one microconduit.

\begin{tabular}{llc}
\hline Site with one microconduit & 'Pinprick Test' sensation & Anesthetized radius $(\mu \mathrm{m})$ \\
\hline Soaked with lidocaine for I minute & Slight sensation & 250 \\
Soaked with lidocaine for 2 minutes & No sensation & 850 \\
Soaked with lidocaine for 3 minutes & No sensation & 1,900 \\
Soaked with lidocaine for 4 minutes & No sensation & 2,200 \\
\hline
\end{tabular}

conduit required 60-90 minutes. Anesthesia became discernable (sensation diminishes perceptibly) after 1 minute. Maximum anesthetic effect and anesthetized radius occurred between 2.5 and 3.5 minutes. The anesthetized radius was $1,800-2,200 \mu \mathrm{m}$. When the lidocaine was applied to the microconduit under a pressure of 30 inches of water, the full anesthetic effect occurred in 1.52 minutes and the anesthetized radius increased to 2,800 $3,000 \mu \mathrm{m}$.

The onset of anesthesia takes longer in microconduits deep enough to yield blood than in shallower, non-blood producing microconduits. Possibly the blood outflow impedes inflow of the externally-applied lidocaine to the sub-stratum corneum tissues, or the clotting blood partially obstructs the microconduit.

\section{Analyte extraction and analysis}

Experiments involving creation of deep microconduits provided blood samples for glucose testing and assessment of the sensation (pain) level involved. Testing was carried out with a FastTake glucose monitor (Lifescan, Milpitas, CA, USA). The instrument's disposable test strips required a minimum blood volume of $1.5 \mu \mathrm{l}$.
These experiments involved one subject using a subjective pain sensation scale of one to ten ('none' to 'sharp'). The test site was always on the side of the left hand fourth finger or the left hand middle finger between the finger end and outer joint. The standard mask-nozzle-generator conditions were used. Opening a microconduit to the blood capillary level consistently took 14-18 seconds. Within 3-6 seconds after removing the scizing device, 1$3 \mu \mathrm{l}$ of blood formed in a drop (Figure 9, left and right). The FastTake test strip end was held against the drop and a sample was drawn into the sensing chamber by capillary action. Results are summarized (Table 4) comparing accessing blood with the Penlet II Automatic Blood Sampler (a lancet system supplied with the FastTake instrument) and through microconduits made by scizing. The tests were done two days apart, with the two lancet tests first, followed by the scizing tests. Time for the lancet test is zero due to its rapid plunge in/withdraw action. Times for scizing were intentionally varied slightly to test sensation and blood flow effects. The glucose readings were all in a 'normal' range. These experiments show that scized microconduits may provide painless acquisition of samples for established blood glucose tests. 

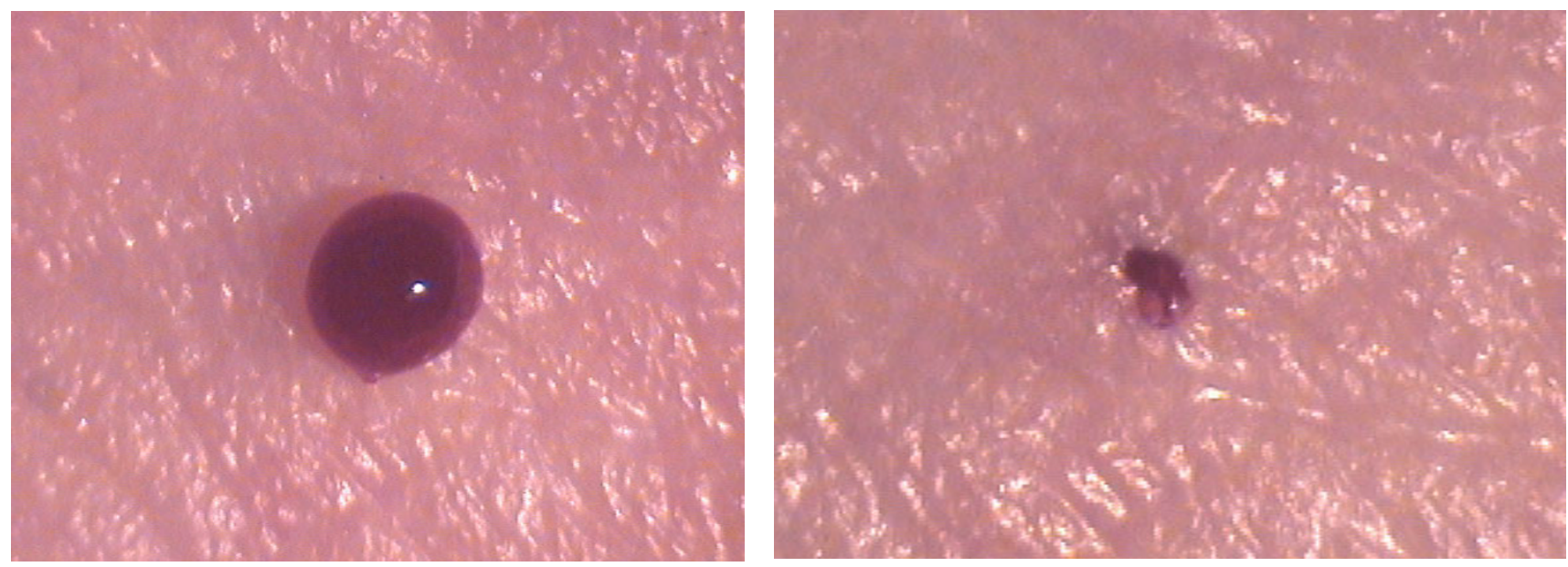

\section{Figure 9}

Blood extraction from microconduit: Left: Blood drop at microconduit (approximately I,600 $\mu$ m in diameter). Right: Blood drop washed off (microconduit approximately $200 \mu \mathrm{m}$ in diameter).

Table 4: Comparison of glucose assay of blood from lanced and microconduit sites.

\begin{tabular}{lcccc}
\hline Technique & Site & Time to blood (s) & $\begin{array}{c}\text { Sensation (I: none, I 0: } \\
\text { sharp) }\end{array}$ & Glucose level (mg/dl) \\
\hline Lancet & Left Ring Finger & 2 & $9-10$ & 95 \\
Lancet & Left Middle Finger & 2 & $8-9$ & 91 \\
Scize & Left Ring Finger & 15 & 2 & 99 \\
Scize & Left Middle Finger & 12 & 1 & Inadequate blood \\
Scize & Left Ring Finger & 18 & 3 & 102 \\
Scize & Left Middle Finger & 16 & 2 & 95
\end{tabular}

\section{Conclusions}

The formation of microconduits in vivo through human stratum corneum, epidermis and dermis, has been demonstrated painlessly and with little or no detectable sensation. These 100-250 $\mu \mathrm{m}$ diameter, $200 \mu \mathrm{m}$ deep openings are made repeatedly, quickly and painlessly through sharp, inert particles microscissioning the tissues. Accurate control of particle size, flux, carrier gas pressure, area exposed to particles, and time of exposure is essential. Microconduit diameter and depth can be controlled; no foreign bodies were discernable after healing. In vivo through-skin drug delivery, analyte access and significantly reduced electrocardiogram electrical impedance have been demonstrated.

\section{Competing interests}

None declared.

\section{List of abbreviations}

N.A., numerical aperture.

\section{Authors' contributions}

JCW, RRA and TOH developed the basic concept. TOH designed and built several experimental microscissioning apparatuses, and carried out the experiments with assistance from TRG, SG and JCW and guidance from RRA. TRG carried out the microscopy and analyzed the images. SG designed and conducted the sensation experiments. TOH, JCW and TRG prepared the manuscript. All authors read and approved the final manuscript.

\section{Acknowledgements}

The authors are grateful to Rieko Tachihara and Milind Rajadhyaksha of the M.G.H Wellman Laboratory of Photomedicine, Department of Dermatology for their assistance and cooperation with in vivo testing, useful discussions and assistance in using the Lucid Vivascope 1000. We thank James Howard of MIT Lincoln Laboratory for the many experiments he per- 
formed in perfecting the scission process. This research was supported by grants from Massachusetts Institute of Technology Lincoln Laboratory, and $\mathrm{NIH}$, with additional support from Massachusetts General Hospital and CIMIT.

\section{References}

I. Owens DR: New horizons - alternative routes for insulin delivery. Nat Rev Drug Discov 2002, I:529-540.

2. Gadsby R: Epidemiology of diabetes. Adv Drug Del Rev 2002, 54: II65-1172

3. Schaefer H, Redelmeier TE: Skin Barrier: Principles of Percutaneous Absorption Karger: Basel; 1996.

4. Langer R: Drug delivery and targeting. Nature 1998, 392 (6679 Suppl):5-10.

5. Kanikkannan N, Kandimalla K, Lamba SS, Singh M: Structure-activity relationship of chemical penetration enhancers in transdermal drug delivery. Curr Med Chem 2000, 7:593-608.

6. Merino V, Lopez A, Hochstrasser D, Guy RH: Noninvasive sampling of phenylalanine by reverse iontophoresis. J Control Release 1999, 61:61-69.

7. Pikal MJ: The role of electroosmotic flow in transdermal iontophoresis. Adv Drug Delivery Rev 200I, 46:28I-305.

8. Potts RO, Tamada JA, Tierney MJ: Glucose monitoring by reverse iontophoresis. Diabetes Metab Res Rev 2002, 18:S49-S53.

9. Prausnitz MR, Bose VG, Langer R, Weaver JC: Electroporation of mammalian skin: A mechanism to enhance transdermal drug delivery. Proc Nat Acad Sci USA 1993, 90:10504-10508.

10. Lee S, Kollias N, McAuliffe DJ, Flotte TJ, Doukas AG: Laser stress waves induce transient increase of the stratum corneum permeability: Implications for transdermal drug delivery. J Invest Dermatol 1997, 108:786.

II. Mitragotri S, Blankschtein D, Langer R: Ultrasound-mediated transdermal protein delivery. Science 1995, 269:850-853.

12. Joshi A, Raje J: Sonicated transdermal drug transport. J Control Release 2002, 83:13-22.

13. Jacques SL, McAuliffe DJ, Blank IH, Parrish JA: Controlled removal of human stratum corneum by a pulsed laser. J Invest Dermatol 1987, 88:88-93.

14. Ilic L, Gowrishankar TR, Vaughan TE, Herndon TO, Weaver JC: Microfabrication of individual $200 \mu \mathrm{m}$ diameter microconduits using high voltage pulsing in salicylic acid and benzoic acid. I Invest Dermatol 200 I, I 1 6:40-49.

15. Henry S, McAllister D, Allen M, Prausnitz M: Microfabricated microneedles: A novel approach to transdermal drug delivery. J Pharm Sci 1998, 87:922-925.

16. Smart WH, Subramanian K: The use of silicon microfabrication technology in painless blood glucose monitoring. Diabetes Technol Ther 2000, 2:549-559.

17. Rajadhyaksha M, Gonzalez S, Zavislan JM, Anderson RR, Webb RH: In vivo confocal scanning laser microscopy of human skin II: Advances in instrumentation and comparison with histology. J Invest Dermatol 1999, I I 3:293-303.

18. Hernández E, González S, González E: Evaluation of topical anesthetics by laser induced sensation: Comparison of EMLA 5\% cream and $\mathbf{4 0 \%}$ lidocaine in an acid mantle ointment. Lasers Surg Med 1998, 23:167-I7I.

\section{Pre-publication history}

The pre-publication history for this paper can be accessed here:

http://www.biomedcentral.com/1741-7015/2/12/prepub 